\title{
Dolphins Underwater Sounds Database: Preliminary Analysis Results
}

\author{
Hristo Zhivomirov ${ }^{1,2}$, Ivaylo Nedelchev ${ }^{1,2}$ \\ ${ }^{1}$ Department of Theory of Electrical Engineering and Measurements, Technical University of Varna, \\ 1 Studentska Str., Varna, Bulgaria \\ ${ }^{2}$ SigNautic Lab - Examination of Underwater Noises, Signals and Vibrations of Marine Vessels and \\ Structures, Technical University of Varna, 1 Studentska Str., Varna, Bulgaria
}

\begin{abstract}
The present paper treats the signal recording and the preliminary analysis of the vocalization of five Caribbean bottlenose dolphins (Tursiops Truncates) in the controlled aquatic environment - a dolphinarium water pool.

The data are taken using measurement equipment from the world's leading companies - Brüel\&Kjær and National Instruments Corporation. All records have been denoised and segmented in advance so that only the vocal-active parts to be analyzed. The most representative part of the records and analysis results are deposited in one of world's most reputable databases - IEEE DataPort ${ }^{\mathrm{Tm}}$, under the name "Dolphins Underwater Sounds Database".

All analyses are conducted in the Matlab ${ }^{\circledR}$ environment, thus they include: signal oscillogram, overall frequency spectrum, time-frequency spectrogram, time-quefrency cepstrogram and also signal correlogram and amplitude histogram. The report is rich in figures that represent some of the most interesting results.

Finally, some common conclusions are drawn, based on the analysis results, and along with considerations for feature work.
\end{abstract}

Keywords - dolphins, underwater, sounds, signals, analysis.

DOI: $10.18421 /$ TEM94-14

https://doi.org/10.18421/TEM94-14

Corresponding author: Hristo Zhivomirov, Department of Theory of Electrical Engineering and Measurements, Technical University of Varna.

Email: hristo_car@abv.bg

Received: 13 August 2020.

Revised: 09 October 2020.

Accepted: 14 October 2020.

Published: 27 November 2020.

(c) BY-NC-ND (C) 2020 Hristo Zhivomirov \& Ivaylo Nedelchev; published by UIKTEN. This work is licensed under the Creative Commons AttributionNonCommercial-NoDerivs 4.0 License.

The article is published with Open Access at www.temjournal.com

\section{Introduction}

The dolphins' intelligence is wide known among the Biology scientists. The dolphins' cognitive abilities are result of highly evolved brain during the evolution. These marine mammals live in groups and families, and they use a complicated set of sounds which represents their own way to establish a social communication in different life-time situations.

These sounds consist of whistles (bursts), clicks and combinations between them in order to form understandable sound (similar to the human speech phonemes), which have specific meaning for the individuals and the group. There is an assumption, which is an object of exploration, that this ensemble of dolphins sounds forms a peculiar language of the species.

Recording, signal processing and analyzing of dolphins' sounds is the key factor for decoding their own language, further understanding of the behavior and the way of life of these marine mammals.

In this paper, the signal recording and preliminary analysis of the vocalization of Caribbean bottlenose dolphins (Tursiops Truncates) in the controlled aquatic environment - a dolphinarium water pool, is considered. The most representative part of the records and analysis results are deposited in the one of world's most reputable database - IEEE DataPort ${ }^{\mathrm{TM}}$, under the name "Dolphins Underwater Sounds Database" [1].

The database contains records of underwater sounds produced by family of five individuals dwelled in "Dolphinarium Festa Varna" (Varna, Bulgaria). The database contains two sets of .wav audio lossless uncompressed files - 13 "bursts" and 104 "clicks" with durations of $250 \mathrm{~ms}$ to $9 \mathrm{~s}$. Each signal is accompanied with results from time, frequency, time-frequency and statistical analysis oscillogram, frequency spectrum, spectrogram, cepstrogram, correlogram, and histogram.

The database includes also another 130 unanalyzed.wav audio segments with duration in the range of $250 \mathrm{~ms}$ to $4 \mathrm{~s}$ and 6 raw unsegmented $5 \mathrm{~min}$ .wav audio records. 


\section{Experimental Design and Data Formats}

The records were made in the autumn 2019 by the "SigNautic Lab" crew, using measurement equipment of the companies Brüel\&Kjær and National Instruments.

The hydroacoustic measurement setup is composed of a few main blocks, as it is shown in Fig. 1:

(i) Hydroacoustic channel, consisted of:

- hydrophone type 8104 [2];

- hydrophone calibrator type 4229 [3];

- hydroacoustic amplifier type Nexus 2692 [4].

(ii) Data acquisition system NI USB-6211 [5].

(iii) Laptop with Matlab ${ }^{\circledR}$ R2019a and specialized data logging Matlab software "Signal Notes" [6].

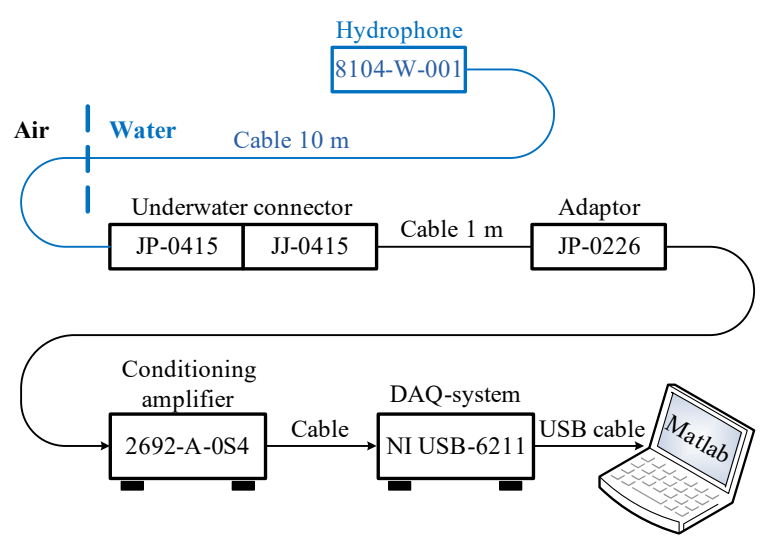

Figure 1. Hydroacoustic measurement setup

The comprehensive list of the parameters of the hydroacoustic channel and the computer-based data acquisition system (DAQ) are given in Tab. 1.

Table 1. Parameters of the measurement setup

\begin{tabular}{|c|c|}
\hline $\begin{array}{l}\text { Hydrophone: } \\
\text { Hydrophone sensitivity: } \\
\text { Hydrophone bandwidth: } \\
\text { Hydrophone depth: } \\
\end{array}$ & $\begin{array}{l}\text { Brüel\&Kjær Type } 8104 \\
-205 \mathrm{~dB} \text { re } 1 \mathrm{~V} / \mu \mathrm{Pa} \\
0 \mathrm{~Hz} \text { to } 120 \mathrm{kHz} \\
0.5 \mathrm{~m}\end{array}$ \\
\hline $\begin{array}{l}\text { Amplifier: } \\
\text { Amplifier sensitivity: } \\
\text { Amplifier bandwidth: }\end{array}$ & $\begin{array}{l}\text { Brüel\&Kjær NEXUS } 2692 \\
10 \mathrm{mV} / \mathrm{Pa} \\
0 \mathrm{~Hz} \text { to } 120 \mathrm{kHz}\end{array}$ \\
\hline $\begin{array}{l}\text { DAQ-system: } \\
\text { DAQ-system input range: } \\
\text { DAQ-system sensitivity: } \\
\text { DAQ-system bandwidth: } \\
\text { DAQ-system sample rate: } \\
\text { DAQ-system resolution: } \\
\text { DAQ-software: }\end{array}$ & $\begin{array}{l}\text { NI USB-6211 } \\
-10 \mathrm{~V} \text { to } 10 \mathrm{~V} \\
91.6 \mu \mathrm{V} \\
0 \mathrm{~Hz} \text { to } 450 \mathrm{kHz} \\
250 \mathrm{kHz} \\
16 \text { bits } \\
\text { Signal Notes }\end{array}$ \\
\hline
\end{tabular}

The records were made in a controlled environment of the main pool of "Dolphinarium Festa Varna" in Varna City, Bulgaria. The pool's geometry and location of the measurement equipment are given in Fig. 2. In Fig. 3, and a part of the measurement setup is depicted.

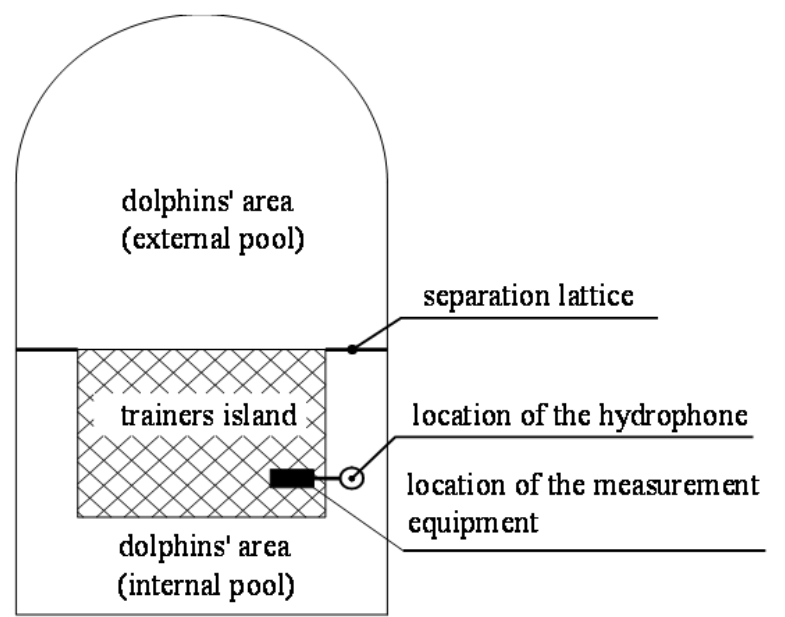

Figure 2. The geometry of the pool and the placement of the hydrophone and the measurement setup

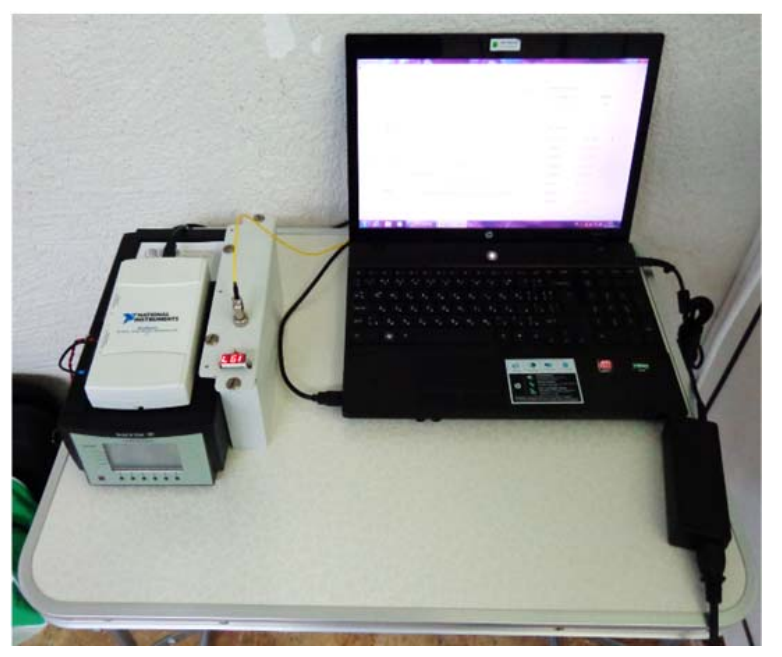

Figure 3. Picture of a part of the measurement setup. One could see the Brüel\&Kjoer NEXUS 2692 conditioning amplifier and the National Instruments DAQ-system NI USB-6211 above it. The battery bank is placed on the right of them along with the laptop. The battery power of the conditioning amplifier allows the $50 \mathrm{~Hz}$ power-line hum to be reduced to a large degree.

Due to the specific frequency band of the dolphins' acoustic signals (up to $120 \mathrm{kHz}$ ), the sampling rate of the DAQ-system was set to be $250 \mathrm{kHz}$ at 16 bit A/D conversion resolution. All records have duration of 5 minutes in order to facilitate the further analysis.

The initial (raw) data has stored as a Matlab ${ }^{\circledR}$ data file (.mat) in single-precision floating-point format to lighten the memory requirements. All records were made in absence of any AC power supply in order to overcome as much as possible the $50 \mathrm{~Hz}$ power-line hum.

Further, after some pre-processing, the data files are converted in uncompressed linear PCM waveform audio (.wav) files with sample rate of 250 $\mathrm{kHz}$ and resolution of 16 bits. The primary analyzes results (oscillograms, spectrograms, cepstrograms, statistics, etc.) are stored as portable network graphic (.png) images and plain text (.txt) data. 


\section{Pre - processing and Analyzing Techniques}

At first, some pre-processing techniques are implemented. Every signal record (as a Matlab ${ }^{\circledR}$ data .mat file) is filtered using a second-order notch digital filter with the notch at frequency of $50 \mathrm{~Hz}$ and a bandwidth of $2 \mathrm{~Hz}$ at the $-3 \mathrm{~dB}$ level in order to defeat any possible power-line artifacts. Further, the signal is normalized to unity peak amplitude, since the absolute underwater sound pressure level is not of interest for our study. Finally, the data record is saved as uncompressed linear PCM waveform audio (.wav) file.

At the second stage, a record segmentation via short-time kurtosis and short-time level crossing-rate estimation is performed, based on the fact, that all vocal-active parts of the record are characterized with higher kurtosis and level-crossing rate in comparison with the silence parts. The kurtosis threshold is set to 4.5 with minimum three crossings above the threshold at 0.05 levels. In addition, all active frames with duration below $250 \mathrm{~ms}$ are neglected. Each active signal frame is saved as separate .wav audio file for further analysis.

The real signal analysis is performed at the third stage. A few signal analysis techniques are implemented in order to reveal the signals' behavior properly. The signals are represented and analyzed in three domains - the classic time and frequency domains and the statistical domain (magnitude statistics and histogram representation), where one is interested in specific signal parameters as max, min, mean and RMS values; dynamic range, crest factor, skewness and kurtosis; signal duration and autocorrelation time.

For revealing the signal behavior into the time domain, two types of signal representation are used classic oscillogram and correlogram. However, a lot more information could be drawn about the signal, using a representation into the frequency domain. As far as the signals under consideration are not stationary (i.e., their frequency content varies by time) the more proper spectrum representation takes place into the time-frequency domain as one-sided spectrogram, which is actually the time-localized one-sided spectrum of the signal. In the same manner a short-time cepstrum (cepstrogram) is computed for revealing of the time-localized harmonic structure of the signal. In order to assist any long-term periodicity detection in the signal, an autocorrelation analysis is implemented and the results of the analysis are given with correlogram. Finally, a histogram is used to reveal the empirical probability density function (PDF) of the analyzed signal and eventually to be classified as some of the known theoretical PDFtypes (e.g., Laplace distribution).

The overall spectrum is computed using the classic periodogram method. The spectrogram is computed using classic short-time Fourier transform and the short-time cepstrogram is computing as the real cepstrum of different partially overlapping signal segments instead of the overall signal. An autocorrelation analysis is also performed, and a correlogram is plotted along with a histogram of the signal. For sake of comparison, the histograms are accompanied with the Laplace superimposed fitted distribution line.

All recorded signals are pre-processed and analyzed in the Matlab $^{\circledR}$ environment using specialdeveloped custom-made scripts for signal analysis, which provides also a proper visualization of the results. The block diagram of the applied signal processing workflow is given in Fig. 4. A comprehensive list of the analysis parameters are listed in Tab. 2.

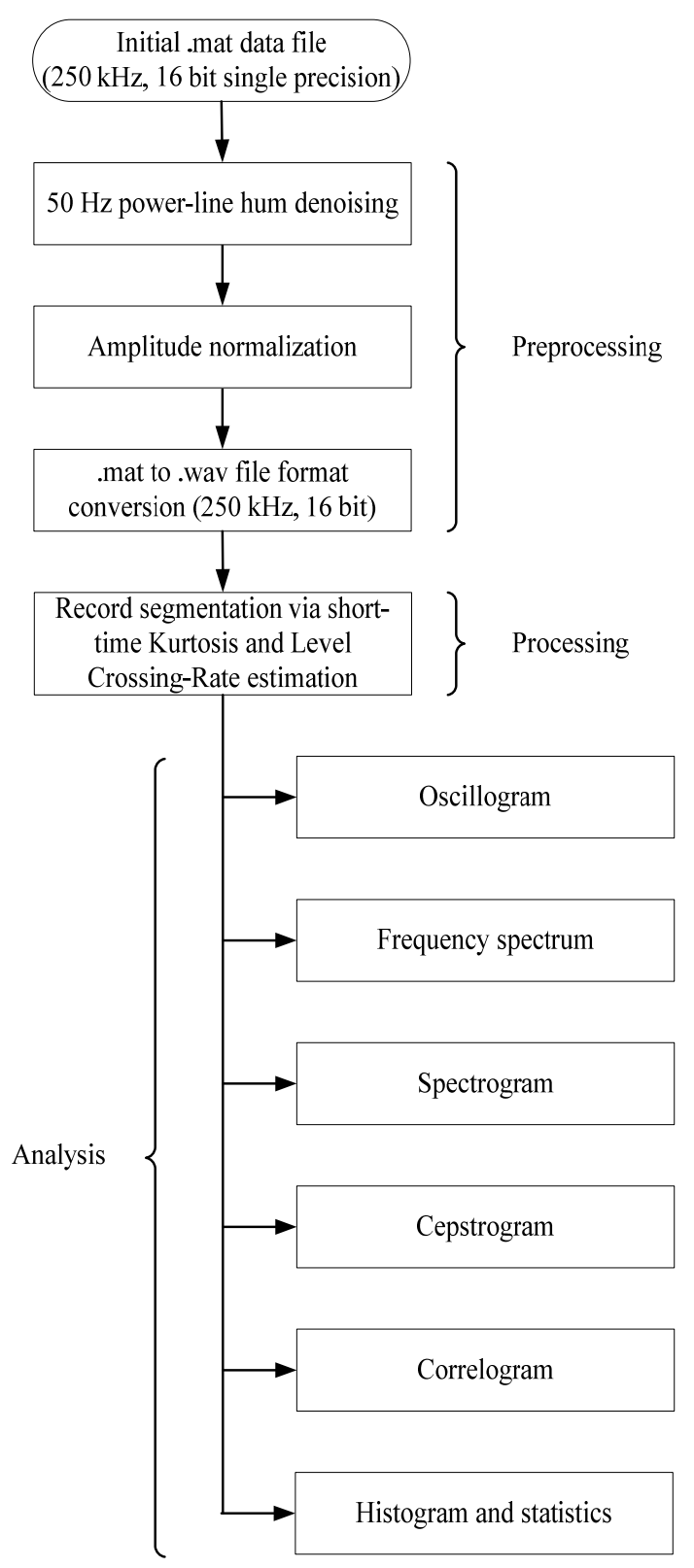

Figure 4. Block diagram of the applied signal processing workflow 
Table 2. Parameters of the signal analysis

\begin{tabular}{||l|l||}
\hline \multicolumn{2}{|c|}{ Spectrum } \\
\hline Window type: & Blackman (periodic) \\
Number of FFT points: & $2^{\wedge}$ nextpow2(signal length) \\
\hline \multicolumn{2}{|c|}{ Spectrogram } \\
\hline Window type: & Blackman (periodic) \\
Window length: & 4096 \\
Hop size: & 512 \\
Number of FFT points: & 32768 \\
\hline \multicolumn{2}{|c|}{ Cepstrogram } \\
\hline Window type: & Hamming (periodic) \\
Window length: & 6144 (for bursts) \\
& 32768 (for clicks) \\
Number of FFT points: & 6144 (for bursts) \\
& 32768 (for clicks) \\
Hop size: & 512 \\
\hline
\end{tabular}

\section{Representative Preliminary Analysis Results}

There are two types of dolphin signals named (by the authors) burst and clicks. The click signals occur very frequently, with duration up to $10 \mathrm{~s}$ and have well distinct amplitude level. The burst signals occur very rarely and have very short duration of about 0.2 $\mathrm{s}$ up to $0.5 \mathrm{~s}$ and very small amplitudes (many times below that of the click signals).

The following notes can be taken as the preliminary analysis results, which are represented in Fig. $5 \div$ Fig. 11:

- the click signals are consisted of pronounced acicular pulse trains which macro and micro structure are given on Fig. $5 \div$ Fig. 8, with their specific time parameters;

- the overall frequency content of the common click signal has specific pattern which is dominated by the first two well pronounced acicular pulses in each period (cf. Figs. 8, 9 and 10);

- the time-localized cepstrum (cepstrogram) of the click signals has two specific parts - two quefrencies around $1 \mathrm{~ms}$ to $10 \mathrm{~ms}$ which are product of the micro-structure of the clicks, and a few variable quefrencies in vicinity of $20 \mathrm{~ms}$ to $60 \mathrm{~ms}$, which are caused by the frequency modulation of the clicks (variable time period between each acicular pulse - see Fig. 11);

Other analysis' results and comments about the bursts and clicks are given in Tab. 3 and Tab. 4, respectively. One may note:

- both clicks and bursts have Laplace PDF;

- in both types of dolphins' signals a strong periodic pattern occurs frequently on the spectrograms. The burst signals have more pronounced harmonic structure which could be seen also in their cepstrograms. It seems like a vowel sound which is typical for humans and other mammals [7].

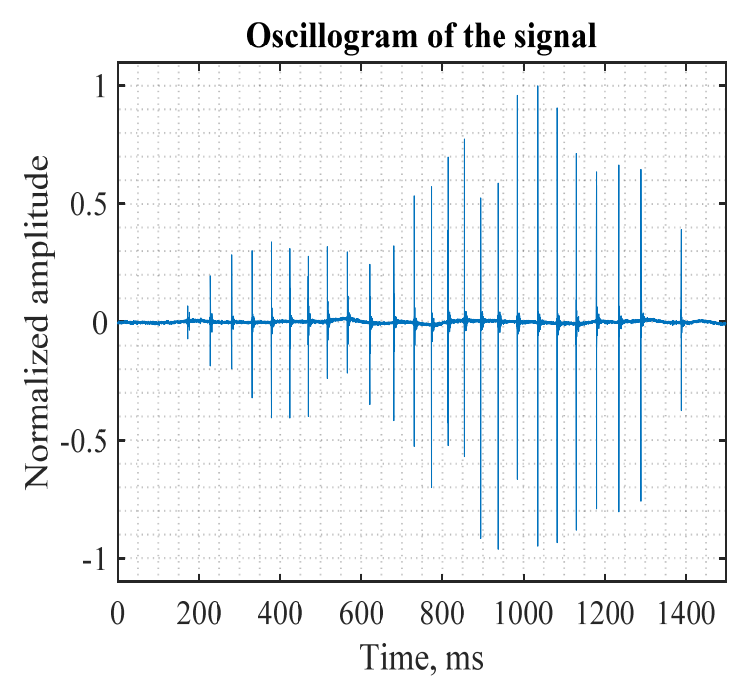

Figure 5. An example of a click signal - an acicular pulse train with pronounced amplitude modulation and slightly variable period. This kind of dolphin's signals is most frequently generated and has duration of about $1 \div 10 \mathrm{~s}$.

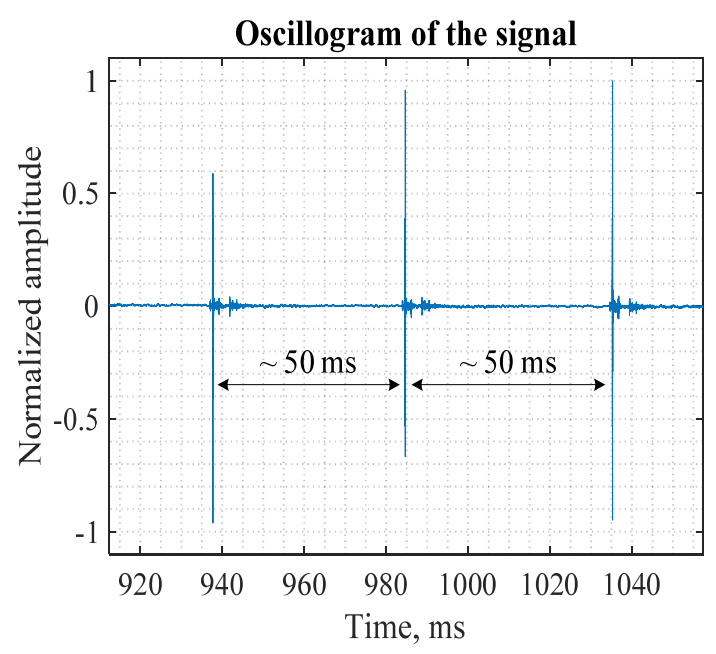

Figure 6. The individual clicks are repeated periodically with period of about $30 \div 60 \mathrm{~ms}$. This can be seen also at the click's cepstrogram in Fig. 11.

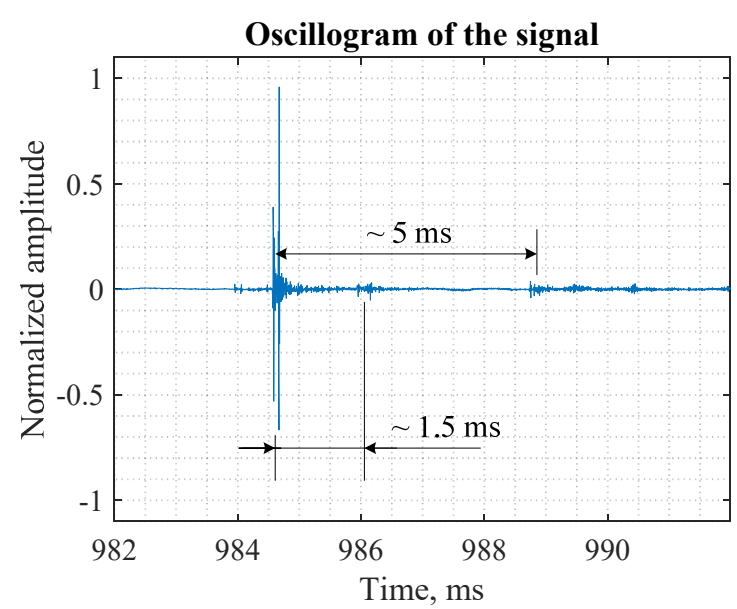

Figure 7. Every individual click has specific structure as is shown - two closely spaced acicular peaks (cf. Fig. 8), and two smaller components at about $1.5 \mathrm{~ms}$ and $5 \mathrm{~ms}$ away. These smaller pulse components have relatively stable time of occurrence (cf. Fig. 11). 


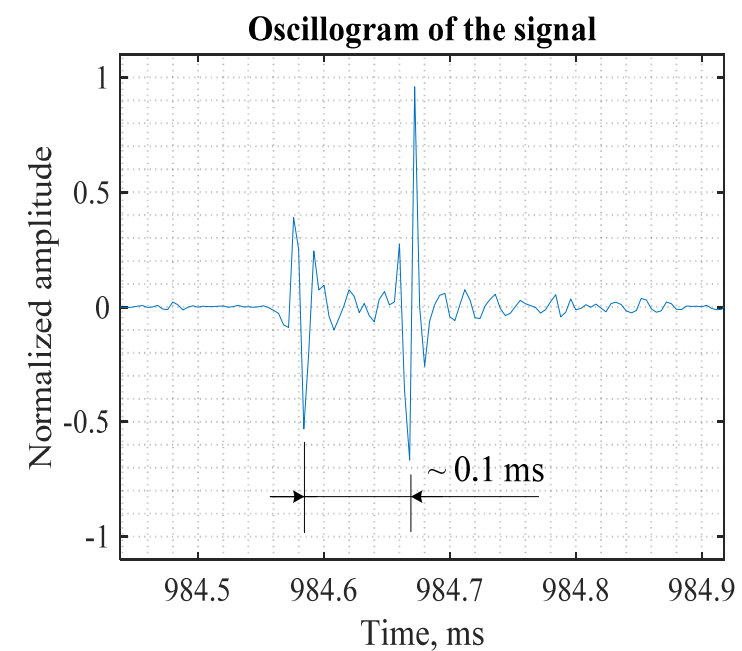

Figure 8. A zoomed view of the two closely spaced acicular peaks as a part of an individual click. The pulses are situated usually at about 0.05 to $0.2 \mathrm{~ms}$ of one another

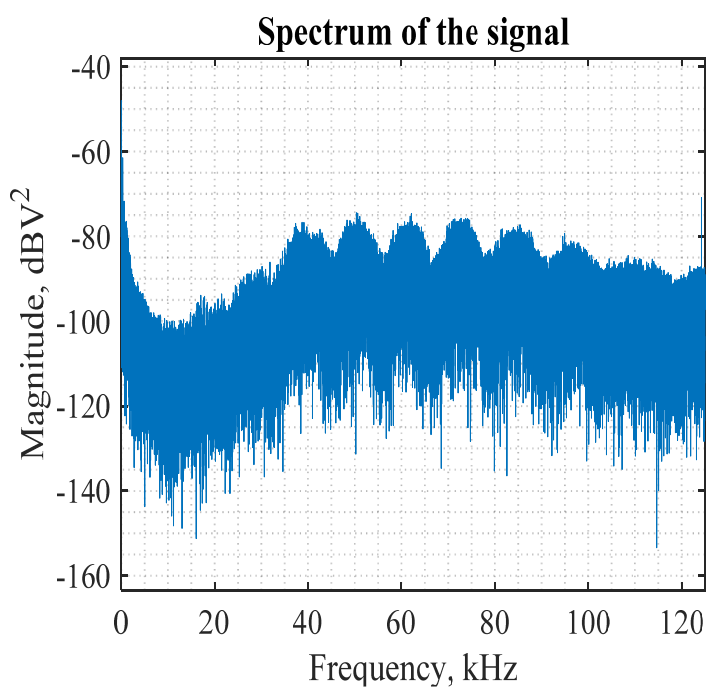

Figure 9. Spectrum of the click signal given in Fig. 5. One could note the spectrum gap between about $5 \mathrm{kHz}$ to 20 $\mathrm{kHz}$ and also the comb-structure caused probably by signal interference.

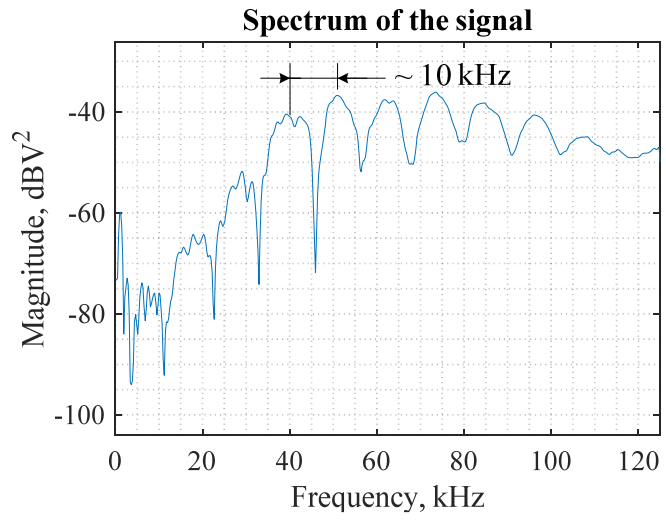

Figure 10. Spectrum of the acicular peaks given in Fig. 8 . It is actually the envelope of the overall spectrum given on the previous figure i.e., the influence of the two acicular peaks and particularly the distance between them is determinative

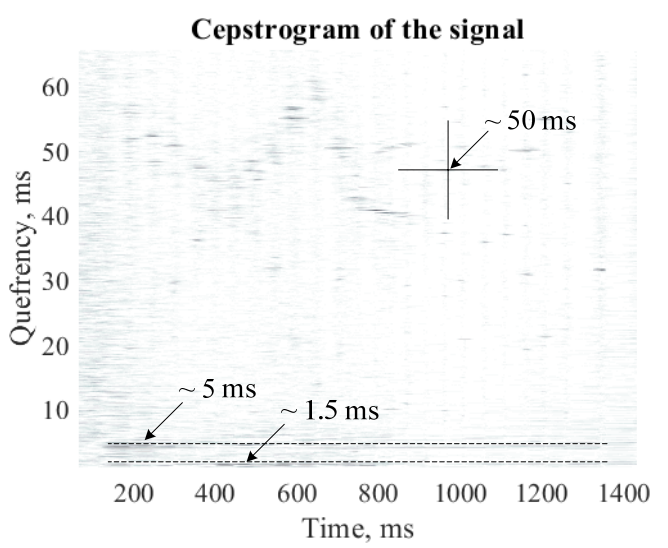

Figure 11. Cepstrogram of the signal given in Fig. 5. One must note: (i) the relatively constant rahmonic at $1.5 \mathrm{~ms}$, and (ii) the rahmonic at $5 \mathrm{~ms}$, corresponding to the timedomain structure at Fig. 7 and (iii) the higher rahmonics

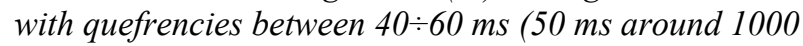
ms timestamp, related with Fig. 6). This higher rahmonics with variable quefrencies attest that there is a kind of frequency modulation along with the amplitude one in the click signal depicted in Fig. 5. 
Table 3. Representative results from the analysis of the burst signals

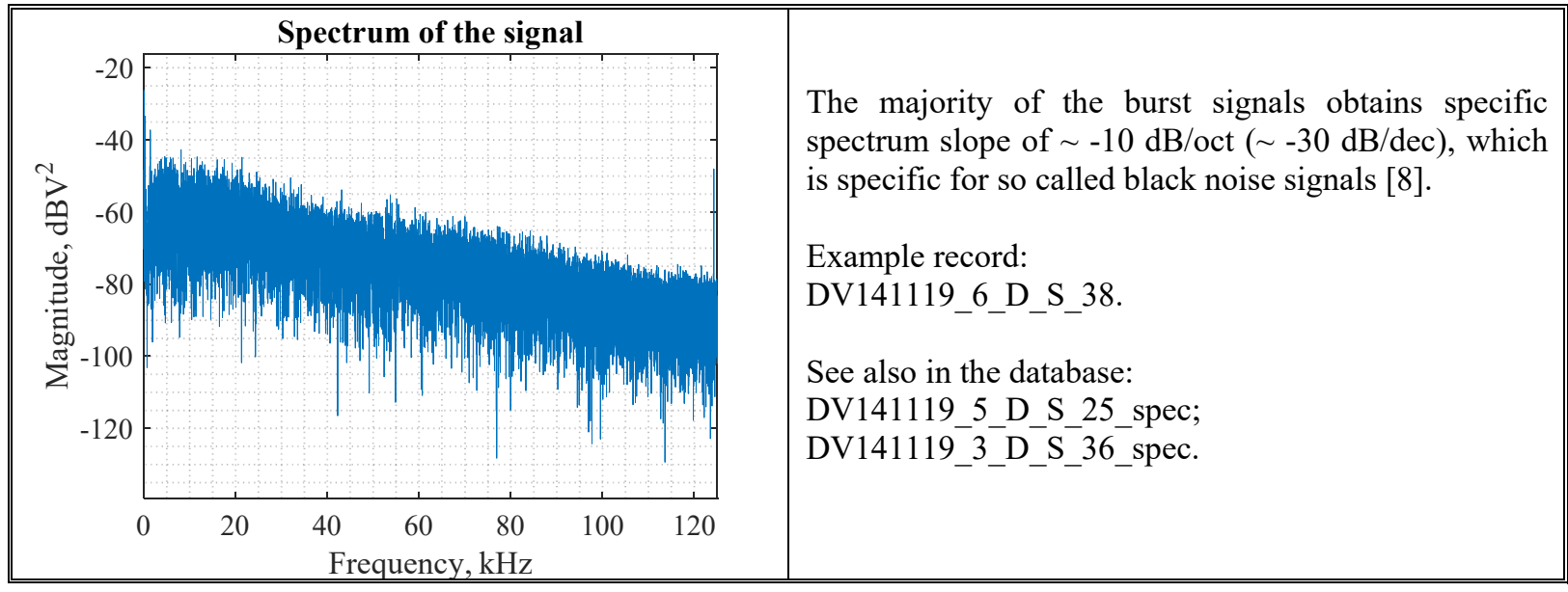

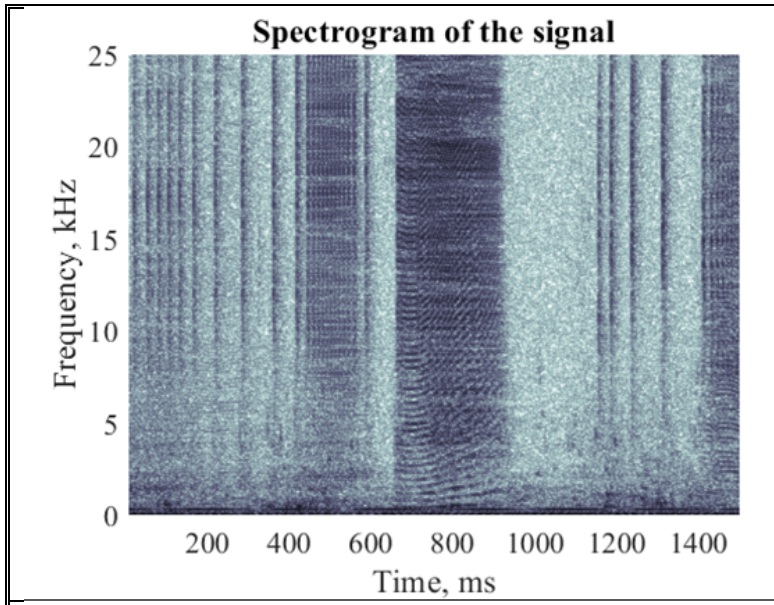

The visual inspection of the signals' spectrograms shows clearly manifested wide-band harmonic structure (see the spectrogram around $800 \mathrm{~ms}$ timestamp). Similar kind of time-frequency domain pattern is specific for the formants in the human speech i.e., a resonance in a spatial volume.

Example record:

DV141119_4_D_S_27.

See also in the database:

DV141119_3_D_S_36_spec_partial;

DV141119_3_D_S_54_spec_partial.

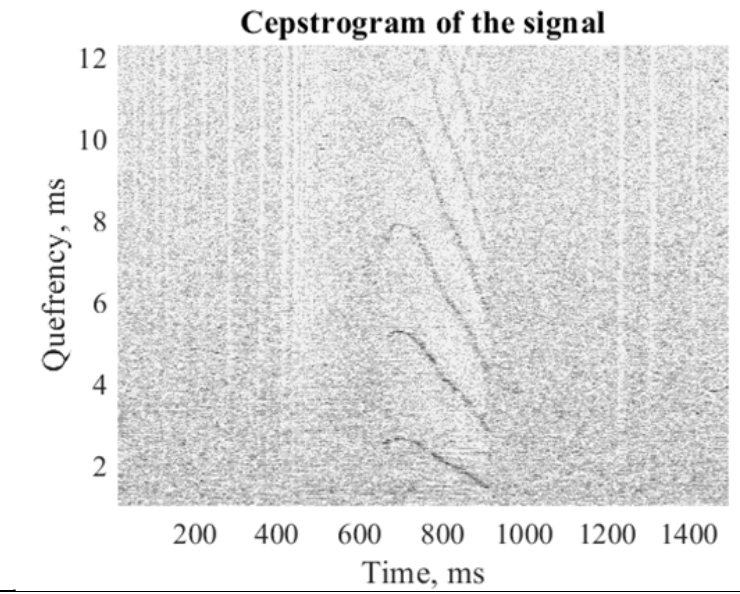

The plot is a typical example of burst's signal cepstrogram. The harmonic structure is evident. Usually, the fundamental quefrency vary in the range of $2 \div 4 \mathrm{~ms}$, with 3 to 6 overtone rahmonics.

Example record:

DV141119_4_D_S_27.

See also in the database:

DV141119_5_D_S_23_ceps;

DV281119_6_D_S_15_ceps.

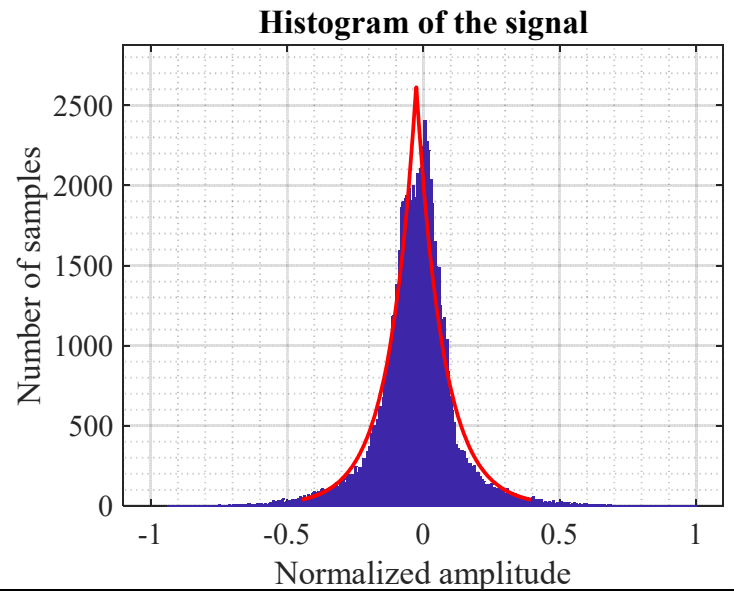

All recorded burst signals obtain Laplace amplitude distribution, similarly to the human speech signals, as is shown in the histogram. Here, the blue bars represent the empirical amplitude distribution, and the red line represents the corresponding theoretical fitted Laplace distribution.

Example record:

DV141119_3_D_S_36.

See also in the database:

DV141119_3_D_S_54 hist;

DV141119_4_D_S_27_hist. 
Table 4. Representative results from the analysis of the click signals
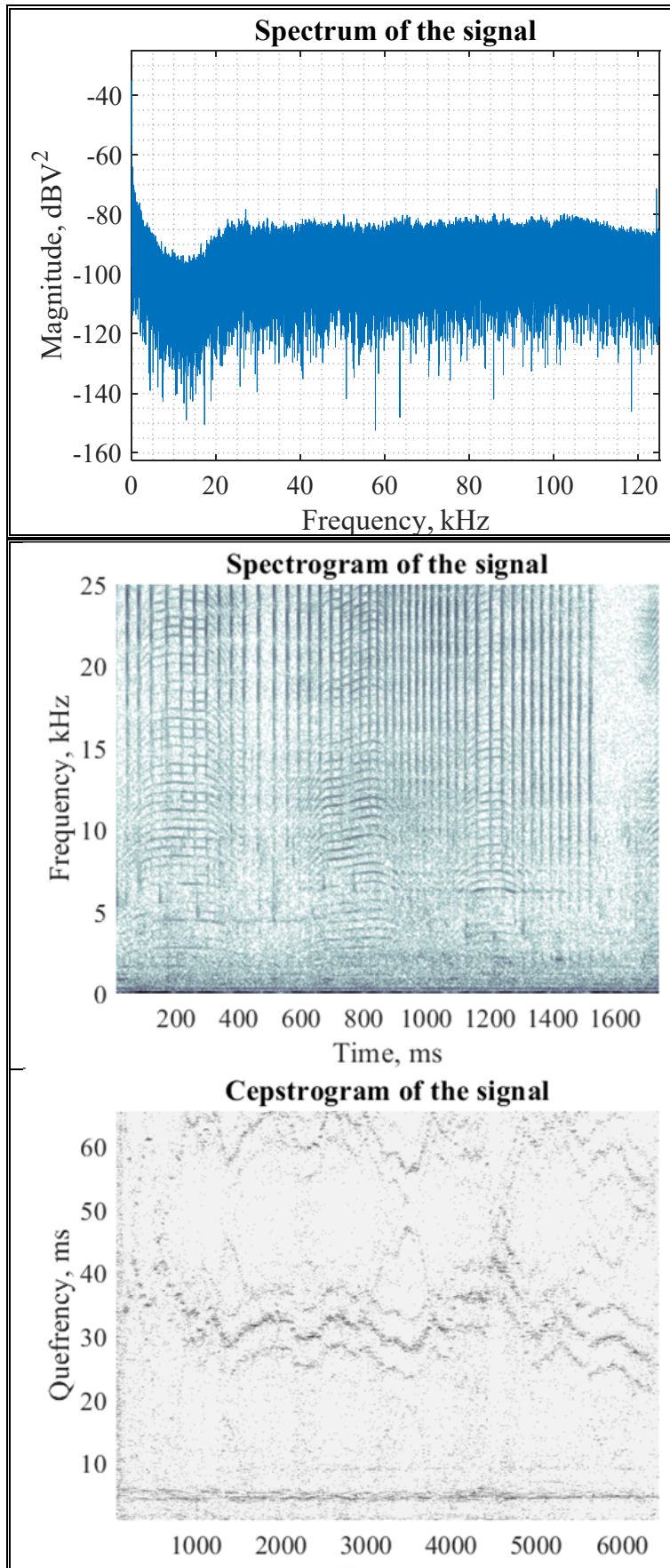

Time, ms

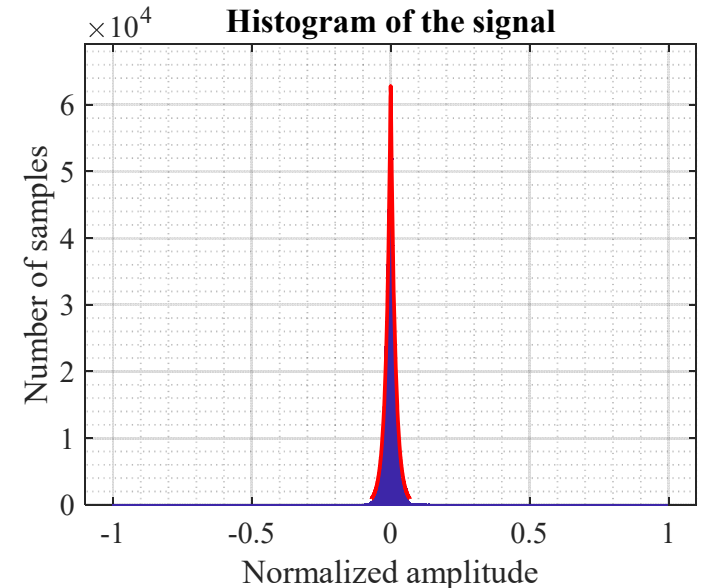

In almost all spectrums of the click signals a gap exists in the range of about $5 \mathrm{kHz}$ to $20 \mathrm{kHz}$.

Example record:

DV281119_1_D_S_41.

See also in the database:

DV281119_1_D_S_51_spec_partial;

DV281119_3_D_S_16_spec_partial.

A clearly manifested wide-band harmonic structure could be seen on some spectrograms. It is interesting that these patterns correspond to the human hearing frequency band. Similar kind of time-frequency domain pattern is detected also in the burst signals. It seems like a vowel sound which is typical for humans and other mammals [7].

Example record:

DV281119_5_D_S_17.

See also in the database:

DV281119_4_D_S_15_spec_partial;

DV281119_6_D_S_29_spec_partial.

A representative example of click signal cepstrogram is shown on the figure. Two rahmonics are of significance - typically around $\sim 5 \mathrm{~ms}$ and $\sim 30 \mathrm{~ms}$. There also rahmonics around $\sim 60 \mathrm{~ms}$ which may be a product of interference (reflection). For majority of cepstrograms the rahmonics are in the vicinity of 20 to $60 \mathrm{~ms}$.

Example record:

DV281119_2_D_S_15.

See also in the database:

DV281119_1_D_S_51_ceps;

DV281119_4_D_S_8_ceps.

Almost all click signals' histograms fitting well the Laplace distribution (similarly to the burst ones). This is specific also for a human speech signals.

Example record:

DV281119_1_D_S_49.

See also in the database:

DV281119_2_D_S_30_hist;

DV281119_2_D_S_39_hist. 


\section{Conclusions}

The study, some representative results which are given in this paper, is a part of a larger forthcoming investigation. The presented results are from a primary exploratory research without attempt for particular concrete conclusions. A further collaboration with biologists and linguists would enlarge the obtained results, using secondary reanalysis of the data already collected in our study, by other researchers.

At this stage a few important results could be point out:

- the dolphin signals have Laplace PDF;

- the click signals have well pronounced amplitude and frequency modulation, which may be a sign of some kind of information carrying;

- burst signals have well pronounced harmonic structure, similar to that of the humans vowel sounds.

The experiments and especially the obtained results are of particular interest for:

- signal processing specialist as far as the analysis places demands for new analysis techniques and approaches concerning the big data analysis (e.g., signal segmentation) and visualization;

- biolinguistic specialist who is interested in the revealing of the dolphins' language and communication;

- cetology specialist, since the obtained results could be bounded to the sound production mechanism and/or the dolphins' behavior.

\section{Acknowledgements}

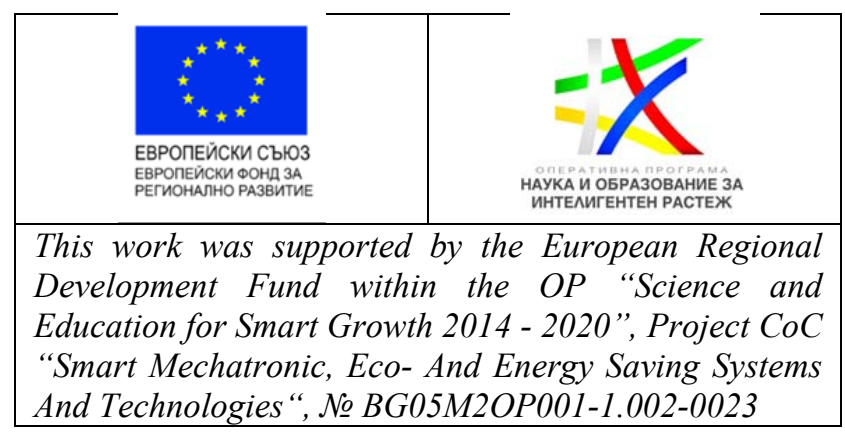

The authors gratefully thank to the company "Festa Dolphinarium Varna” Ltd., without whose support our research study would not have been possible.

\section{References}

[1]. Zhivomirov, H., Nedelchev, I., Dimitrov, G. (2020). Dolphins Underwater Sounds Database. New York, IEEE Dataport.

DOI: http://dx.doi.org/10.21227/n00y-kq67

[2]. Brüel \& Kjaer. (2019). Product Data - Hydrophones Types 8103, 8104, 8105, 8106. Naerum, Brüel \& Kjaer.

[3]. Brüel \& Kjaer. (2006). Product Data - Hydrophone Calibrator Type 4229. Naerum, Brüel \& Kjaer.

[4]. Brüel \& Kjaer. (2015). Product Data - The NEXUS Range of Conditioning Amplifiers Types 2690, 2691, 2692 and 2693. Naerum, Brüel \& Kjaer.

[5]. National Instruments. (2017). Specifications of NI USB-6211. Austin, National Instruments Corp.

Retrieved from:

https://www.ni.com/pdf/manuals/375195d.pdf [accessed: 20 May 2020].

[6]. Zhivomirov, H. (2020). Data Acquisition and Logging with Matlab (open-source software for data acquisition and logging with NI USB-600x, USB-621x and USB -65xx DAQ hardware). Natick, Matlab Central File Exchange. Retrieved from: https://www.mathworks.com/matlabcentral/fileexchan ge/68476-data-acquisition-and-logging-with-matlabimplementation [accessed: 10 August 2020].

[7]. Pisanski, K., Cartei, V., McGettigan, C., Raine, J., \& Reby, D. (2016). Voice modulation: a window into the origins of human vocal control?. Trends in cognitive sciences, 20(4), 304-318.

DOI: $10.1016 / j . t i c s .2016 .01 .002$

[8]. Schroeder, M. (2009). Fractals, chaos, power laws: Minutes from an infinite paradise. Courier Corporation. 
Table A.1. Generalized information about the "Dolphins Underwater Sounds Database"

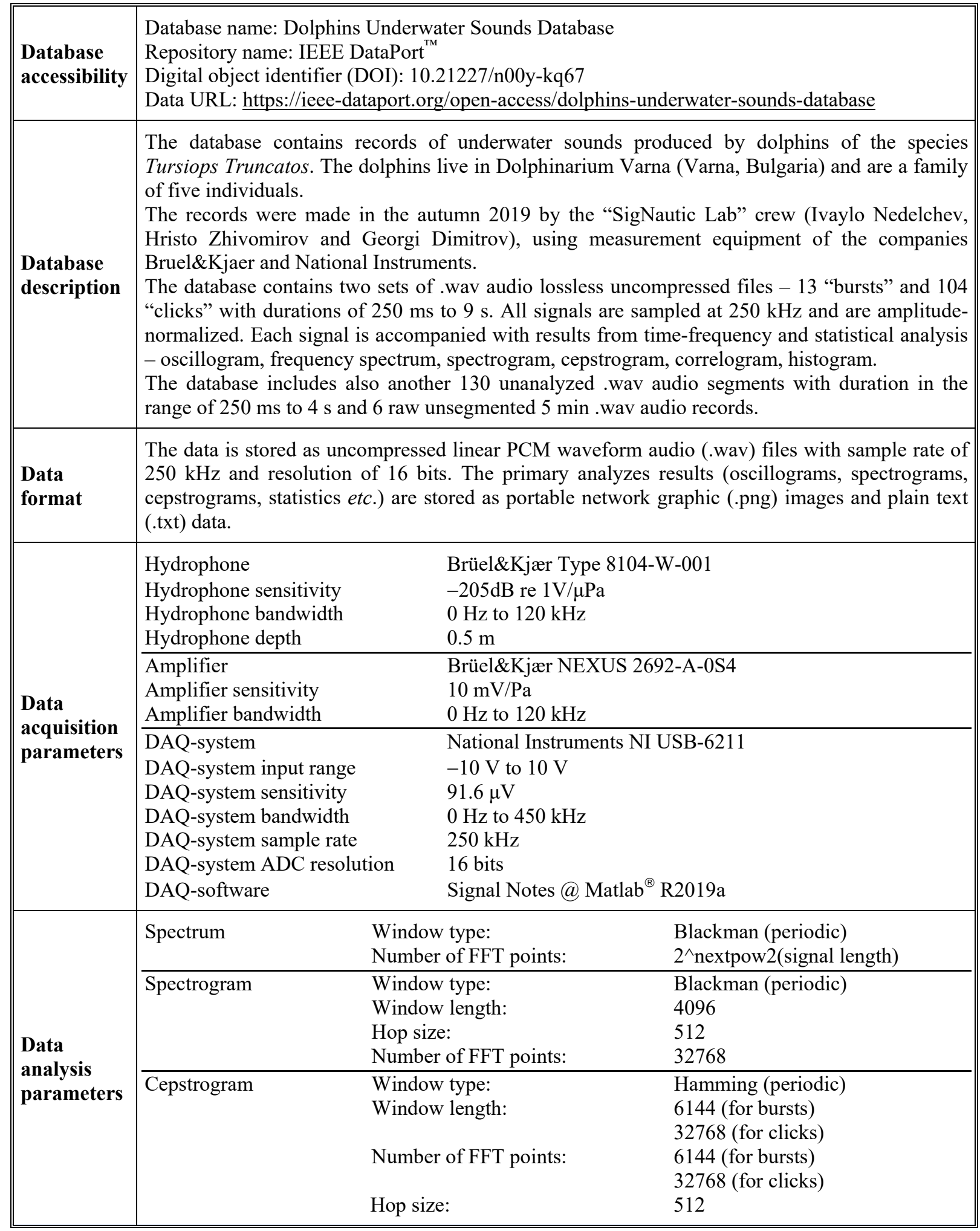

